\title{
Identification of a DO white dwarf and a PG 1159 star in the ESO SN la progenitor survey (SPY) ${ }^{\star}$
}

\author{
K. Werner ${ }^{1}$, T. Rauch ${ }^{1,2}$, R. Napiwotzki ${ }^{3}$, N. Christlieb ${ }^{4}$, D. Reimers ${ }^{4}$, and C. A. Karl ${ }^{2}$ \\ ${ }^{1}$ Institut für Astronomie und Astrophysik, Universität Tübingen, Sand 1, 72076 Tübingen, Germany \\ e-mail: werner@astro.uni-tuebingen.de \\ ${ }^{2}$ Dr.-Remeis-Sternwarte, Universität Erlangen-Nürnberg, Sternwartstraße 7, 96049 Bamberg, Germany \\ 3 Department of Physics and Astronomy, University of Leicester, University Road, Leicester, LE1 7RH, UK \\ ${ }^{4}$ Hamburger Sternwarte, Universität Hamburg, Gojenbergsweg 112, 21029 Hamburg, Germany
}

Received 24 April 2004 / Accepted 18 May 2004

\begin{abstract}
We present high-resolution VLT spectra of a new helium-rich DO white dwarf (HE1314+0018) and a new PG 1159 star (HE1429-1209), which we identified in the ESO SPY survey. We performed NLTE model atmosphere analyses and found that the PG 1159 star is a low-gravity, extremely hot $\left(T_{\text {eff }}=160000 \mathrm{~K}, \log g=6\right)$ star, having a carbon-helium dominated atmosphere with considerable amounts of oxygen and neon $(\mathrm{He}=38 \%, \mathrm{C}=54 \%, \mathrm{O}=6 \%, \mathrm{Ne}=2 \%$ by mass $)$. It is located within the planetary nebula nuclei instability strip, hence, future searches for an associated PN as well as for stellar pulsations might be successful. The DO white dwarf is a unique object. From the relative strength of neutral and ionized helium lines we found $T_{\text {eff }} \approx 60000 \mathrm{~K}$, however, the He II lines are extraordinarily strong and cannot be fitted by any model. Similar problems were encountered with hot subdwarfs and white dwarfs showing signatures of a super-hot wind. The reason is unknown.
\end{abstract}

Key words. stars: abundances - stars: evolution - stars: AGB and post-AGB - stars: white dwarfs stars: individual: HE1429-1209 - stars: individual: HE1314+0018

\section{Introduction}

The ESO Supernovae Ia Progenitor Survey (SPY) is aimed at finding binary white dwarfs to test the double-degenerate (DD) scenario for supernova type Ia progenitors (Napiwotzki et al. 2001, 2003). Multi-epoch high-resolution spectroscopy of almost 1000 white dwarfs has been obtained with the UV-Visual Echelle Spectrograph (UVES; Dekker et al. 2000) mounted at the UT2 telescope (Kueyen) of the ESO VLT, allowing identification of DDs by radial velocity variations.

Other interesting objects found in the SPY project are, e.g., subdwarf B stars (Lisker et al. 2004), and DB white dwarfs (Christlieb et al. 2004, in prep.). Here we report on the identification and spectrum analysis of a PG 1159 star and a DO white dwarf.

PG 1159 stars are hot hydrogen-deficient (pre-) white dwarfs $\left(T_{\text {eff }}=75000-200000 \mathrm{~K}, \log g=5.5-8\left[\mathrm{~cm} / \mathrm{s}^{2}\right]\right.$; Werner 2001). They are perhaps the outcome of a late heliumshell flash, a phenomenon that drives the currently observed fast evolutionary rates of three well-known objects (FG Sge, Sakurai's object, V605 Aql). Flash-induced envelope mixing produces a H-deficient stellar surface. The photospheric composition then essentially reflects that of the region between

* Based on observations at the Paranal Observatory of the European Southern Observatory for programs No. 165.H-0588(A) and 167.D-0407(A). the $\mathrm{H}$ - and He-burning shells in the precursor AGB star. The He-shell flash transforms the star back to an AGB star and the subsequent, second post-AGB evolution explains the existence of Wolf-Rayet central stars of planetary nebulae and their successors, the PG 1159 stars.

DO white dwarfs are hot helium-rich white dwarfs $\left(T_{\text {eff }}=47500-120000 \mathrm{~K}, \log g=7-8\right.$; Dreizler \& Werner 1996), which are thought to be the progeny of PG 1159 stars. After entering the white dwarf (WD) cooling sequence, heavy elements are removed from the PG 1159 atmospheres by gravitational settling when the luminosity decreases during their evolution, leaving behind the lightest element, helium, and transforming the star into a DO white dwarf.

Although this evolutionary scenario seems to be well established, many unsolved problems remain, which justifies a closer look at new PG 1159 stars and DO white dwarfs. They are relatively rare objects when compared to the total number of white dwarfs and central stars known, often with quite remarkable individual characteristics.

We present here a newly discovered He-rich white dwarf with unusually strong spectral lines. We also present a new PG 1159 star which belongs to the rare subgroup of highly luminous objects. In Sect. 2 we describe the observations and then turn to the spectral analysis of the two stars in Sect. 3. We conclude with a discussion in Sect. 4. 


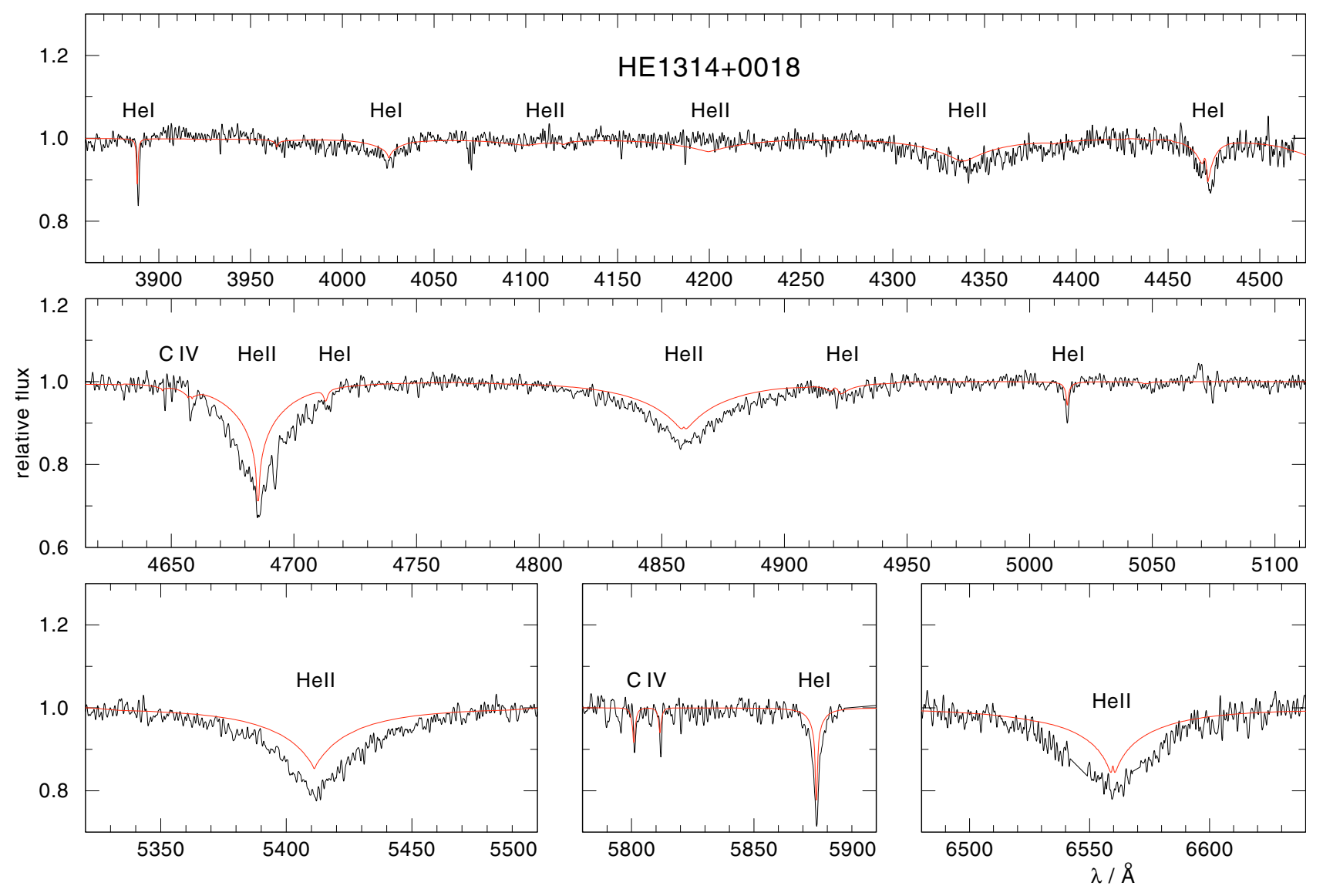

Fig. 1. Spectrum of the new DO white dwarf. Overplotted is a model with $T_{\text {eff }}=60000 \mathrm{~K}, \log g=7.5$, and $\mathrm{C} / \mathrm{He}=0.001$. The He I lines fit well, but all the He in lines in the model are much too weak. No acceptable fit to the complete spectrum is found. The synthetic spectrum was degraded to the instrumental resolution.

\section{Observations}

$\operatorname{HE} 1314+0018\left(\alpha_{2000}=13^{\mathrm{h}} 17^{\mathrm{m}} 24^{\mathrm{s}} .74, \delta_{2000}=+00^{\circ} 02^{\prime} 37^{\prime \prime} .1\right.$, $B=15.6 \mathrm{mag})$ and HE1429-1209 $\left(\alpha_{2000}=14^{\mathrm{h}} 32^{\mathrm{m}} 20^{\mathrm{s}} .69\right.$, $\delta_{2000}=-12^{\circ} 22^{\prime} 47^{\prime \prime} .9, B=15.8 \mathrm{mag}$ ) were identified in the Hamburg ESO survey (HES; Wisotzki et al. 2000; Christlieb et al. 2001) as white dwarf candidates and were therefore included in the SPY project. HE1314+0018 (=PG 1314+003) was classified as "sdOC" in the Palomar-Green catalog (Green et al. 1986). Based on the SPY spectra presented here, HE1314+0018 is listed in Christlieb et al. (2001) as DO white dwarf. Later, it was also identified in the Sloan Digital Sky Survey (Krzesiński et al. 2004). HE1429-1209, independently re-discovered by us, was classified by Kilkenny et al. (1997) as "cont" or "sdO?" (see their Table 1). The EC survey identifier of this object is EC 14296-1209. The high-resolution UVES spectra obtained in the course of the SPY project revealed that HE1429-1209 is actually a PG 1159 star.

The spectra presented here were taken between April 2000 and July 2002. The integration time for each spectrum was $10 \mathrm{~min}$. HE1314+0018 was observed three times and HE1429-1209 twice. The respective spectra were co-added to improve the $\mathrm{S} / \mathrm{N}$ ratio. The SPY instrument setup (Dichroic 1, central wavelengths $3900 \AA$ and $5640 \AA$ ) used UVES in a dichroic mode and nearly complete spectral coverage from $3200 \AA$ to $6650 \AA$ with only two $\approx 80 \AA$ wide gaps at $4580 \AA$ and $5640 \AA$ is achieved. SPY was implemented as a service mode program, which took advantage of those observing conditions, which are not usable by most other programs (moon, poor seeing, clouds). A wide slit (2'. 1$)$ is used to minimize slit losses and a $2 \times 2$ binning is applied to the CCDs to reduce read-out noise. The wide slit reduces the spectral resolution to $R=18500(0.2 \AA$ at $3600 \AA)$ or better, if seeing disks were smaller than the slit width.

The spectra were reduced with a procedure developed by Karl (2004, in prep.) using the ESO MIDAS software package, partly based on routines from the UVES pipeline reduction software provided by ESO (Ballester et al. 2003). Since the sampling of the spectra is much higher than needed for our purpose, we rebinned them to $0.1 \AA$ stepsize. This produces only a slight degradation of the resolution while considerably improving the signal-to-noise level. Figures 1 and 3 display the spectra of the DO white dwarf and the PG 1159 star, respectively.

\section{Spectral analysis}

Line blanketed non-LTE model atmospheres were computed using our PRO2 code (Werner et al. 2003). The models assume plane-parallel geometry and hydrostatic and radiative equilibrium.

The atomic models employed for the calculation of PG 1159 stellar atmosphere models account for the most 


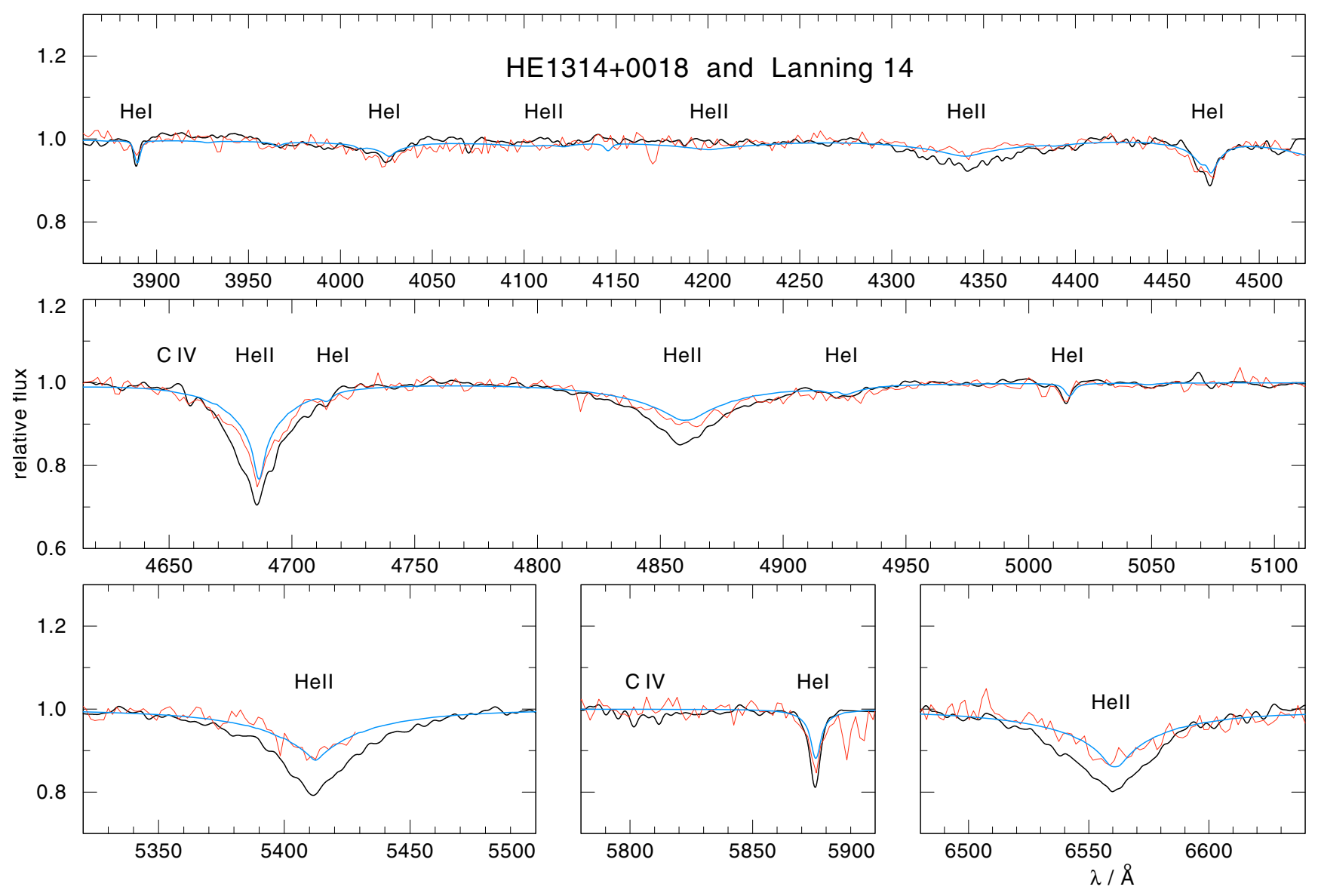

Fig. 2. HE1314+0018 (thick line) compared to Lanning 14 (thin line), which is a DO with similar parameters. The smooth thin line is a model atmosphere with $T_{\text {eff }}=60000 \mathrm{~K}$ and $\log g=8$ and fits well to Lanning 14 ( $\left.T_{\text {eff }}=58000 \mathrm{~K}, \log g=7.9\right)$. The He II lines in HE1314+0018 are significantly deeper and cannot be fitted with any model spectrum. The HE1314+0018 spectrum and the model spectrum have been degraded according to the resolution of the Lanning 14 spectrum $(3 \AA)$.

abundant elements in PG 1159 stars, namely, helium, carbon, oxygen, and neon. For more details we refer to Werner et al. (2004a,b). The atomic models for DO atmospheres comprise very detailed $\mathrm{He}$ I and $\mathrm{He}$ II ions. Essentially, the input physics of the models is similar to that described in Dreizler \& Werner (1996). The model atom for the calculation of carbon line profiles in the DO is similar to that used for the PG 1159 star and is consistently included in the model structure calculations.

\subsection{The DO star HE1314+0018}

The spectrum of HE1314+0018 exhibits lines from neutral and ionized helium, i.e. we have detected a "cool" DO ("hot" DOs show only He II lines). This usually allows an accurate determination of $T_{\text {eff }}$ and $\log g$ (Dreizler \& Werner 1996). We also detect the $\mathrm{C}_{\text {IV }} 5801 / 5812 \AA$ doublet and a very weak feature of this ion close to He II $4686 \AA$. A first guess for the photospheric parameters can be obtained by comparison with a compilation of many other DO spectra in the reference just mentioned. Based on this, we computed a small grid of pure helium models with $T_{\text {eff }}=55000-80000 \mathrm{~K}$ and $\log g=7-9$. The $\mathrm{C}$ Iv lines are indicative for a $\mathrm{C}$ abundance of the order $0.1 \%$ by number.
Surprisingly, we are unable to obtain an acceptable fit to the observed spectrum. Figure 1 shows the best fit model to the He I lines, which is obtained with $T_{\text {eff }}=60000 \mathrm{~K}$ and $\log g=7.5$. It is obvious that the synthetic He II lines are much too weak compared to the observation. We found no model that can fit the strong He II lines. If we increase $T_{\text {eff }}$, these lines become slightly deeper, but never reach the observed equivalent widths. $T_{\text {eff }}>70000 \mathrm{~K}$ can be excluded because the He I lines disappear. $T_{\text {eff }}<55000 \mathrm{~K}$ is excluded because the He I lines become too strong and the He II lines are getting even weaker. A compromise is reached at about $65000 \mathrm{~K}$ in a sense that the relative $\mathrm{He} \mathrm{I} / \mathrm{He}$ II line strengths in the model are similar to the observed ones. But, clearly, lines from both ionization stages are much too weak. This becomes also evident if we compare the spectrum of HE1314+0018 directly to that of other DOs in this temperature range (e.g. in Dreizler \& Werner 1996). In Fig. 2 a comparison to Lanning 14 is shown. This DO has parameters very similar to our new DO.

As already indicated, this situation was never encountered before in analyses of cool DOs. However, this problem is reminiscent of what we faced with those "hot" DOs that show signatures of a super-hot wind. We have found that a large fraction $(50 \%)$ of the hot DO white dwarfs shows such signatures in the optical spectrum (Werner etal. 1995; 


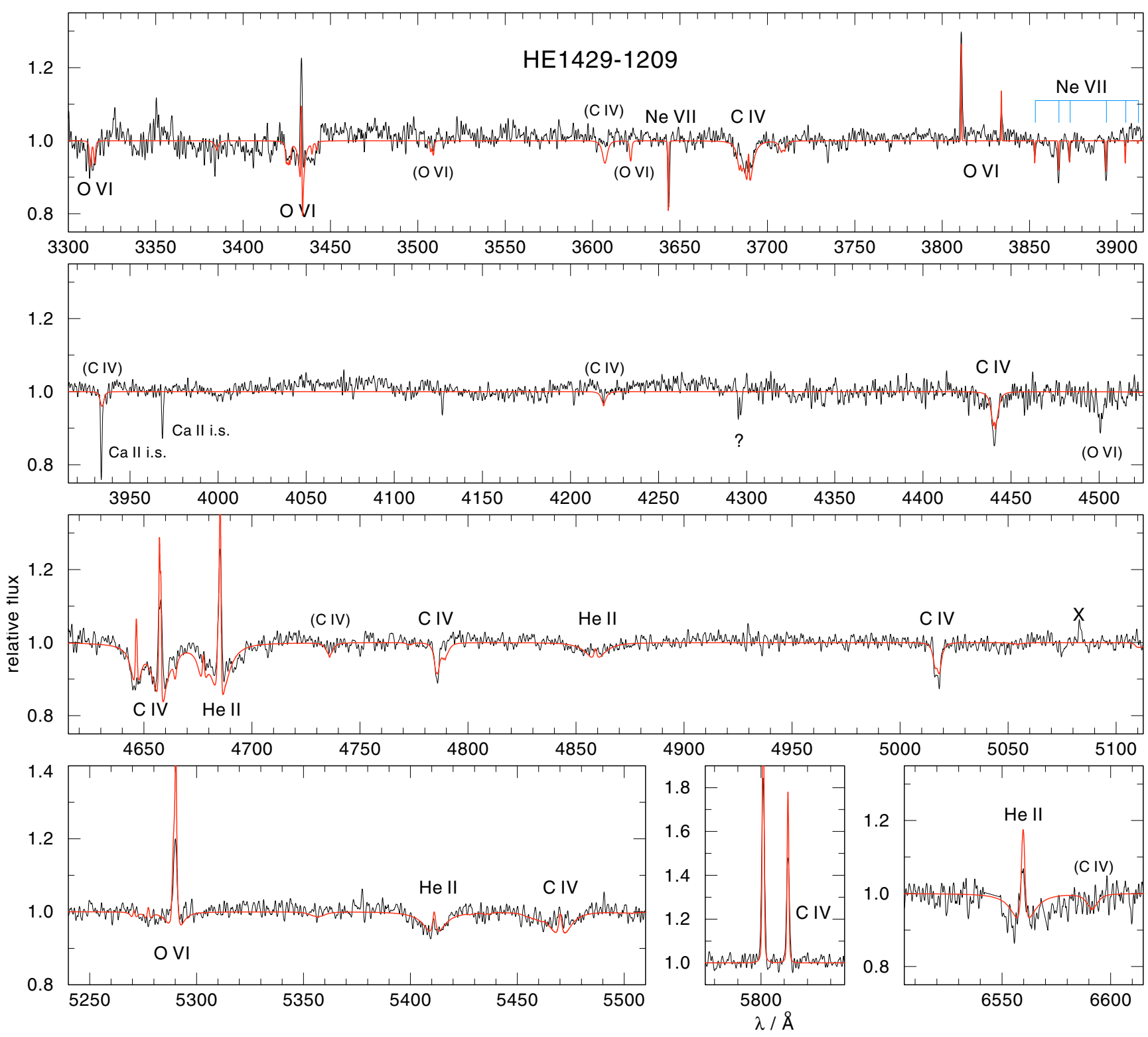

Fig. 3. Spectrum of the new PG 1159 star with the best fit model overplotted. An expanded view of the 3627-3662 A and 3808-3914 $\AA$ regions comprising neon lines are shown in Figs. 4 and 5. "X" denotes an artifact. There is an unidentified absorption line at $4295 \AA$. The observed spectrum was smoothed with a Savitzky-Golay filter (Press et al. 1992) and the synthetic spectrum was degraded to the instrumental resolution.

Dreizler et al. 1995a). These hot stars also show high ionization absorption lines of the CNO elements (e.g. C VI, N vII, $\mathrm{O}$ viII, and even $\mathrm{Ne} x$ ). The high excitation potentials involved require temperatures approaching almost $10^{6} \mathrm{~K}$ and the triangular shaped line profiles suggest their formation in a rapidly accelerating wind from the WD, reaching a terminal velocity of the order of $10000 \mathrm{~km} \mathrm{~s}^{-1}$. At the same time, all He II lines have symmetric profiles and, like in the present case, they are much too strong to be fitted by any DO model. The reason for this simultaneous occurrence of "hot-wind" signatures and toostrong He II lines in hot DOs is unknown.

Our new DO star also has too strong He II lines but, in contrast, shows no signatures of super-high ionization metal lines. But on the other hand these signatures are rather weak in some of the "hot-wind" DOs. So we can speculate that in the present case the physical reason for the excessive line strengths is the same like in the "hot-wind" DOs, but the respective super-high ionization metal lines remain undetected here. The low effective temperature of HE1314+0018 in comparison to the "hotwind" DOs might be linked to this non-detection.

We stress that this wind phenomenon along with too-strong lines is not restricted to DO white dwarfs. We have also detected one DA white dwarf with similar high-ionization features (Dreizler etal. 1995a) and much too deep hydrogen Balmer lines when compared to models. This is the only case discovered among DA white dwarfs so far, and the question arises why there is such a strong preference among DOs to show this phenomenon.

A similar problem with inconsistent line-profile fits was encountered in analyses of some $\mathrm{sdB}$ and sdOB stars, in the 


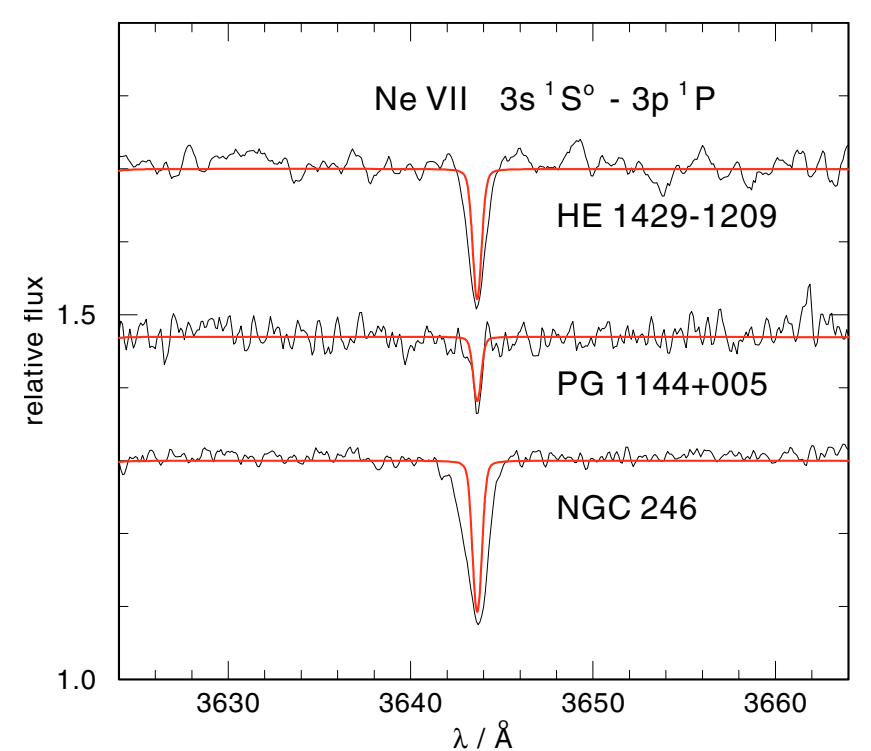

Fig. 4. Detection of the Ne VII $3644 \AA$ singlet in HE1429-1209. Overplotted is our best fit model with a Ne abundance set to $2 \%$ by mass. For comparison, we show spectra of two other PG 1159 stars together with their models (assuming also $\mathrm{Ne}=2 \%$; presented in Werner et al. 2004b).

range $T_{\text {eff }}=40000-60000 \mathrm{~K}$ and $\log g \approx 6$ (Heber et al. 1999). The temperature derived from the $\mathrm{He}$ I/He II ionization balance does not fit the strength of the hydrogen Balmer lines. The Balmer lines are too strong, in analogy to our case presented here, where we have too strong He II lines. The Balmer lines in these subdwarfs can be fitted by models with $T_{\text {eff }}$ lower than derived from the $\mathrm{He} \mathrm{I} / \mathrm{He}$ II ionization balance, whereas in contrast the He II lines in our DO white dwarf in tendency require a higher $T_{\text {eff. }}$. Whether the solution to these problems with inconsistent line-profile fits in white dwarfs and subdwarfs can be explained by strong line blanketing (e.g. by iron-group elements) remains to be investigated.

We have also considered the possibility that HE1314+0018 shows a binary composite spectrum. This can be ruled out because, as already mentioned, the strong He II lines cannot be matched by any DO model, even if we disregard the presence of He I lines.

From the $\mathrm{C}_{\mathrm{IV}} 5801 / 5812 \AA$ doublet we find a carbon abundance of $\mathrm{C} / \mathrm{He}=0.003$ by number, under the assumption of $T_{\text {eff }}=60000 \mathrm{~K}$ and $\log g=8$.

\subsection{The PG 1159 star HE1429-1209}

A comparison of the HE1429-1209 spectrum with other PG 1159 stars immediately reveals that we have found another very hot, low-gravity PG 1159 star which is rather similar to the well known central star of the planetary nebula NGC 246 and the central star RX J2117.1+3412 (Rauch \& Werner 1997). The latter has $T_{\text {eff }}=170000 \mathrm{~K}$ and $\log g=6.0$ (Werner et al. 1996). According to the classification scheme introduced by Werner (1992), the spectral subtype is "lgE" (meaning lowgravity star with emission lines). The spectrum is characterized by lines from highly ionized species (He II, C Iv, O vi, Fig. 3), often present as absorption lines with central emission reversals or even as pure emission lines. The absorption features in "lgE" subtypes are narrower than in other PG 1159 stars pointing at a lower surface gravity, hence, higher stellar luminosity.

We have calculated a small grid of model atmospheres in the range $140-180 \mathrm{kK}$ and $\log g=5.7-6.5$, assuming an element composition of $\mathrm{He}=38 \%, \mathrm{C}=54 \%, \mathrm{O}=6 \%$, $\mathrm{Ne}=2 \%$ (by mass). This is the composition found for RXJ2117.1+3412. Having found the best fit model for HE1429-1209, we vary the element abundances, guided by comparison of selected lines in the observed and synthetic spectra. No attempt is made to perform a more formal fit, because that would require an extensive model grid varying six parameters. Our error estimates are conservative in order to account for this approach.

We stress in particular the presence of a Ne viI line at $3644 \AA$ (Fig. 4). We have recently performed a systematic investigation about neon in a large number of PG 1159 stars (Werner et al. 2004b) and found that such a prominent Ne viI line is preferentially exhibited by "lgE" subtypes. It has been shown that neon is strongly overabundant (about 20 times solar, i.e., $2 \%$ by mass), otherwise the line would be undetectable. The strength of this line is similar to that in NGC 246, confirming that the neon abundance in HE1429-1209 is also of the order $2 \%$. In the paper just cited, we have identified for the first time a Ne viI multiplet in the 3850-3910 range. Figure 5 shows that this multiplet is also present in our new PG 1159 star. Generally, the computed equivalent widths of the Ne VII lines in HE1429-1209 appear underestimated, as it is the case in NGC 246, suggesting that the neon abundance may be even higher than $2 \%$.

Our best fitting model essentially confirms our first guess for the atmospheric parameters. We find:

$$
\begin{aligned}
& T_{\text {eff }}=160000 \mathrm{~K} \pm 15000 \mathrm{~K} \quad \log g=6.0 \pm 0.3\left[\mathrm{~cm} / \mathrm{s}^{2}\right] \\
& \mathrm{He}=38 \% \quad \mathrm{C}=54 \% \quad \mathrm{O}=6 \% \quad \mathrm{Ne}=2 \% \text {. }
\end{aligned}
$$

The estimated error for abundances is 0.3 dex. Stellar mass and luminosity can be derived by comparing the star's position in the $\log g-\log T_{\text {eff }}$ diagram with theoretical evolutionary tracks. We use the post-AGB He-burner tracks of Wood \& Faulkner (1986; Fig. 6) and derive:

$$
\begin{aligned}
& M / M_{\odot}=0.68_{-0.08}^{+0.15} \quad \log L / L_{\odot}=4.04_{-0.05}^{+0.09} \\
& d / \mathrm{kpc}=5.2_{-2.2}^{+1.6}
\end{aligned}
$$

HE1429-1209 is still very luminous, i.e., on the horizontal part of the post-AGB evolutionary track in the HRD, located within the domain of the hottest central stars of planetary nebulae. The spectroscopic distance was obtained by comparing the visual flux ( $V=16.1 \mathrm{mag}$ ) with the flux of the final model: $\left.\mathrm{H}_{v}[5400 \AA]=2.85 \times 10^{-3} \mathrm{erg} / \mathrm{cm}^{2} / \mathrm{s} / \mathrm{Hz}\right)$. Interstellar reddening is probably small and was neglected for this determination.

\section{Conclusions}

The new He-rich white dwarf joins the group of 33 DO white dwarfs (see Dreizler \& Werner 1996 for an analysis of most of them). It belongs to the subgroup of "cool" DO stars which 


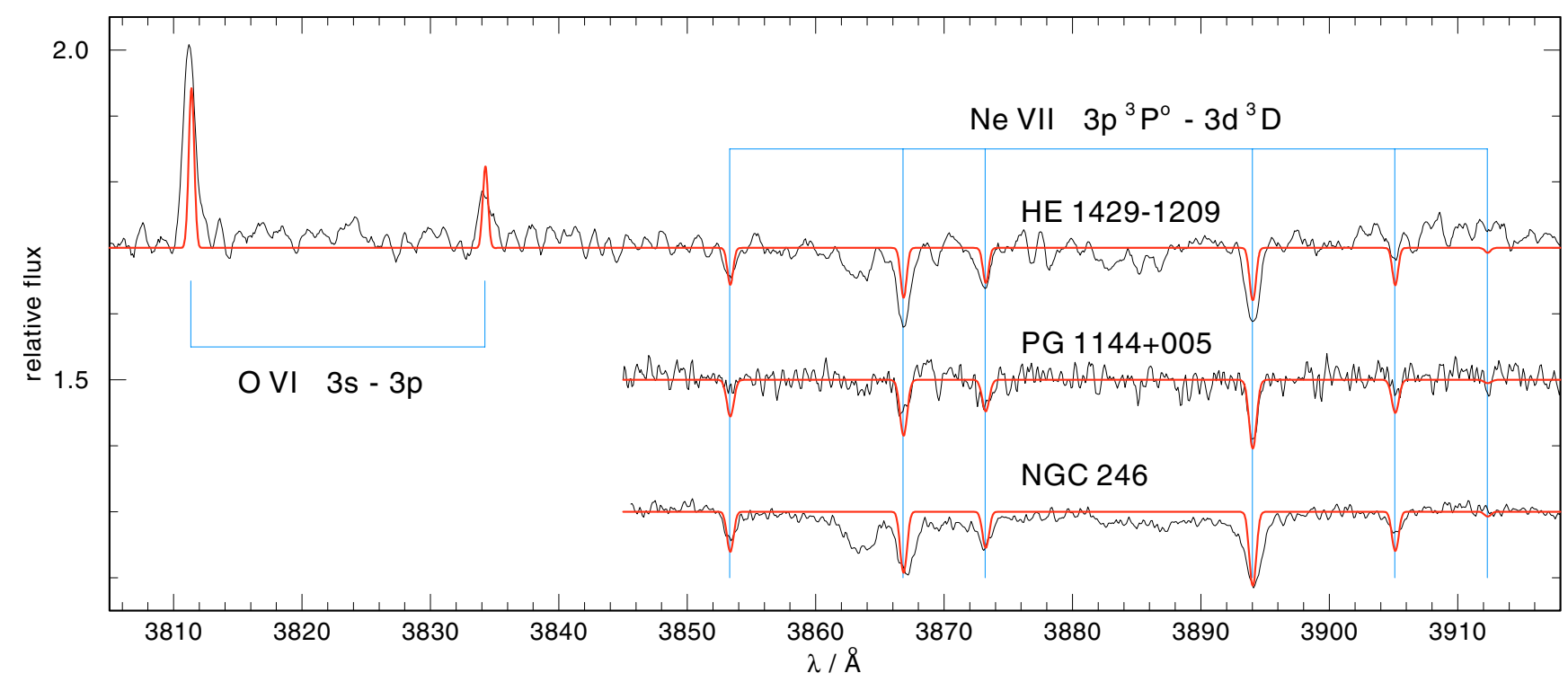

Fig. 5. Detection of a Ne vII multiplet in HE1429-1209. For comparison, we show spectra of two other PG 1159 stars. Synthetic spectra are from the same models as in Fig. 4. There is an unidentified absorption line at $3862 \AA$.

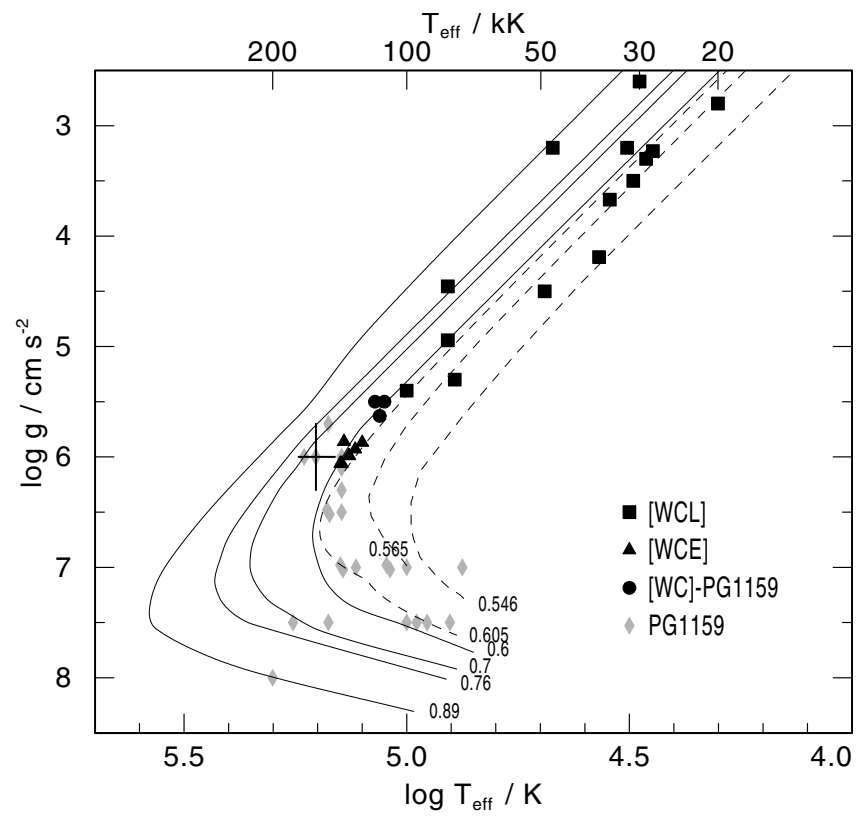

Fig. 6. Position of the new PG 1159 star HE1429-1209 (with error bars) in the $\log g-\log T_{\text {eff }}$ diagram. We identify Wolf-Rayet central stars (Hamann 1997), PG 1159 stars as well as [WC]-PG 1159 transition objects. Evolutionary tracks are from Schönberner (1983) and Blöcker (1995) (dashed lines), and Wood \& Faulkner (1986) (labels: mass in $\left.M_{\odot}\right)$. For details on other objects than HE1429-1209 see Werner (2001).

exhibit both ionized and neutral helium lines. In contrast to all other cool DOs, HE1314+0018 shows extraordinarily strong He II lines, which cannot be matched by any model atmosphere. This phenomenon was only known from the "hot" DOs (i.e. those exhibiting exclusively He II lines). However, in these cases it simultaneously occurs along with ultra-high ionization metal lines, probably stemming from a super-hot wind. No such signatures are seen in HE1314+0018. The extreme strength of the He II lines is not understood.
The effective temperature of HE1314+0018 is close to $60000 \mathrm{~K}$. We found $\mathrm{C} / \mathrm{He}=0.003$ by number. This means that HE1314+0018 is the coolest DO with a safe detection of a photospheric trace metal. The occurrence of metals in DOs is thought to be radiatively supported against gravitational settling. HE1314+0018 is obviously located at the cool end of the $T_{\text {eff-range, beyond which radiation forces can no longer keep }}$ carbon up in the atmosphere. This provides a constraint for detailed self-consistent NLTE diffusion models for DO white dwarfs, which still need to be computed.

The new PG 1159 star is among the hottest objects within this group, which now comprises 32 stars (30 of them are listed in Dreizler et al. 1995b). It is very similar to six members (having subtype $\operatorname{lgE}$ ), which are low-gravity, i.e., highluminosity objects, being still on the hot, horizontal part of their post-AGB evolutionary track in the HRD. It is thus not surprising that all except one of these previously known $\operatorname{lgE}$ PG 1159 stars have an associated planetary nebula, which are rather old and large, but not yet dispersed as is the case for most of the more evolved PG 1159 stars (having entered the white dwarf cooling sequence). So we expect that our new PG 1159 star could also be surrounded by an extended PN. This prompted us to perform a search for faint nebulosities on the Palomar Observatory Sky Survey (POSS) plates and the Southern H-Alpha Sky Survey Atlas (SHASSA; Gaustad et al. 2001). We could not find anything resembling a PN on the POSS plates, but an elongated faint nebulosity, extending over several degrees, is visible in the SHASSA image. However, the structure is not centered on HE1429-1209. It is possible that this is the HE1429-1209 PN in a very advanced stage of interaction with the interstellar medium, but deeper images would be necessary to confirm this. In the $\log g-T_{\text {eff }}$ diagram (Fig. 6) HE1429-1209 is located close to the PNNVs (PN nuclei variables) K1-16, RX J2117.1+3412, NGC 246, and Longmore 4. It could well be, that a photometric study will show that the new PG 1159 star is also a pulsator. Concerning the atmospheric 
abundances, HE1429-1209 is a "typical" PG 1159 star. It further confirms that these objects present intershell matter of the progenitor AGB star on their surface, possibly as a result of a late He-shell flash.

Acknowledgements. We thank Ronald Weinberger (Innsbruck) for helpful discussions. T.R. is supported by the DLR under grant 50 OR 0201. R.N. acknowledges support by a PPARC advanced fellowship. C.K. gratefully acknowledges financial support by the Deutsche Forschungsgemeinschaft through grant NA365/2-2. We are grateful to the ESO staff at Paranal for carrying out the VLT/UVES Service Mode observations. This article made use of the Southern H-Alpha Sky Survey Atlas (SHASSA), which is supported by the National Science Foundation.

\section{References}

Ballester, P., Boitquin, O., Modigliani, A., et al. 2003, UVES Pipeline User's Manual, ESO, Garching

Blöcker, T. 1995, A\&A, 299, 755

Christlieb, N., Wisotzki, L., Reimers, D., et al. 2001, 366, 898

Dekker, H., D’Odorico, S., Kaufer, A., Delabre, B., \& Kotzlowski, H., Proc. SPIE, 4008, 534

Dreizler, S., \& Werner, K. 1996, A\&A, 314, 217

Dreizler, S., Heber, U., Napiwotzki, R., \& Hagen, H.-J. 1995a, A\&A, 303, L53

Dreizler, S., Werner, K., \& Heber, U. 1995b, in White Dwarfs, ed. D. Koester, \& K. Werner, Lect. Notes Phys., 443 (Springer: Berlin), 160

Gaustad, J. E., McCullough, P. R., Rosing, W., \& van Buren, D. 2001, PASP, 113, 1326

Green, R. F., Schmidt, M., \& Liebert, J. 1986, ApJS, 61, 305
Hamann, W.-R. 1997, in Planetary Nebulae, ed. H. J. Habing, \& H. J. G. L. M. Lamers (Kluwer), IAU Symp., 180, 91

Heber, U., Edelmann, H., Lemke, M., \& Napiwotzki, R. 1999, in White Dwarfs, ed. J.-E. Solheim, \& E. G. Meistas, ASP Conf. Ser., 169,551

Kilkenny, D., O’Donoghue, D., Koen, C., Stobie, R. S., \& Chen, A. 1997, MNRAS, 287, 867

Krzesiński, J., Nitta, A., Kleinman, S. J., et al. 2004, A\&A, 417, 1093

Lisker, T., Heber, U., Napiwotzki, R., et al. 2004, A\&A, submitted

Napiwotzki, R., Christlieb, N., Drechsel, D., et al. 2001, AN, 322, 411

Napiwotzki, R., Christlieb, N., Drechsel, D., et al. 2003, ESO Messenger, 112, 25

Press, W. H., Teukolsky, S. A., Vetterling, W. T., \& Flannery, B. P. 1992, Numerical Recipes, 2nd ed. (Cambridge University Press)

Rauch, T., \& Werner, K. 1997, in The third Conf. on faint blue stars, ed. A. G. D. Philip, J. Liebert, \& R. A. Saffer (New York: L. Davis Press, Schenectady), 217

Schönberner, D. 1983, ApJ, 272, 708

Werner, K. 1992, in Atmospheres of early-type stars, ed. U. Heber, \& C. S. Jeffery (Springer, Berlin) Lect. Notes Phys., 401, 273

Werner, K. 2001, in Low mass Wolf-Rayet stars: Origin and evolution, ed. T. Blöcker, L. B. F. M. Waters, \& A. A. Zijlstra, Ap\&SS, 275, 27

Werner, K., Dreizler, S., Heber, U., et al. 1995, A\&A, 293, L75

Werner, K., Dreizler, S., Heber, U., et al. 1996, A\&A, 307, 860

Werner, K., Deetjen, J. L., Dreizler, S., et al. 2003, in Stellar atmosphere modeling, ed. I. Hubeny, D. Mihalas, \& K. Werner, ASP Conf. Ser., 288, 31

Werner, K., Rauch, T., Barstow, M. A., \& Kruk, J. W. 2004a, A\&A, 421, 1169

Werner, K., Rauch, T., Reiff, E., Kruk, J. W., \& Napiwotzki, R. 2004b, A\&A, submitted

Wisotzki, L., Christlieb, N., Bade, N., et al. 2000, A\&A, 358, 77

Wood, P. R., \& Faulkner, D. J. 1986, ApJ, 307, 659 